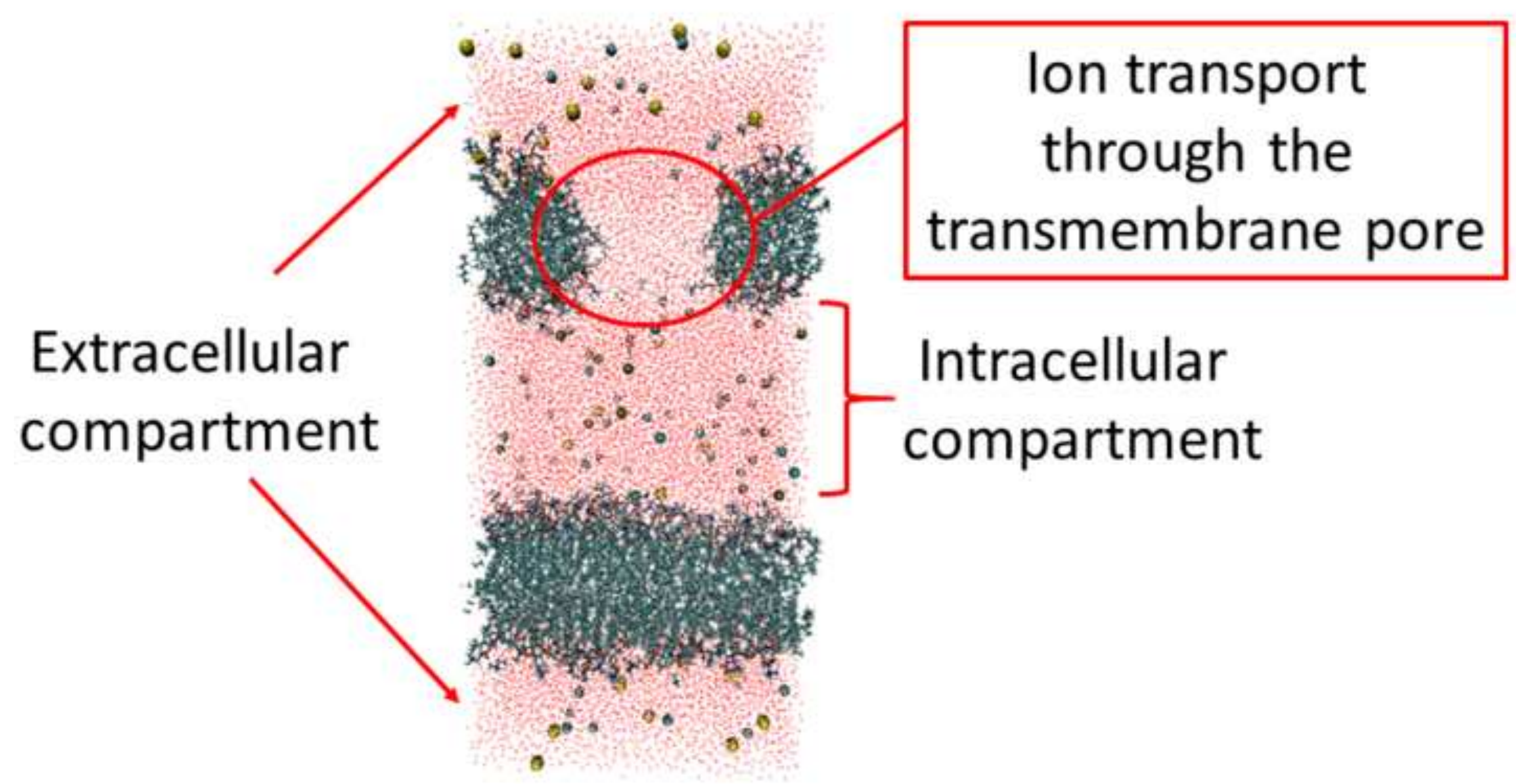




\title{
Mechanism resulting in chemical imbalance due to cellular damage associated with mechanoporation: A molecular dynamics study
}

\author{
Yelena R. Sliozberg ${ }^{\ddagger}$,* and Tanya L. Chantawansri ${ }^{\dagger}$ \\ ${ }^{\dagger}$ U.S. Army Research Laboratory, Aberdeen Proving Ground, MD 21005, USA \\ \$KC Global Inc, Aberdeen Proving Ground, MD 21005, USA \\ " corresponding author, email: yelena.r.sliozberg.ctr@mail.mil, postal address: 4600 Deer \\ Creek Loop, Aberdeen Proving Ground, MD 21009, telephone: 410-306-0700
}

\begin{abstract}
To elucidate the mechanism of ion transport through a transmembrane pore, all-atom molecular dynamics simulations were employed. A model membrane where a pore connects the intra- and extra-cellular compartment was considered. Pores with radii of $1.5 \mathrm{~nm}$ or less exhibited resealing over the course of $135 \mathrm{~ns}$ simulations, and ionic disturbance is minimal. Ion transport through a larger pore ( $2 \mathrm{~nm}$ radius) leads to a substantial change in the intra- and extra-cellular ionic concentrations. The influx of $\mathrm{Na}^{+}$and $\mathrm{Cl}^{-}$ions down their concentration gradients is greater than the efflux of $\mathrm{K}^{+}$leading to an osmotic influx of water.
\end{abstract}

Keywords: pore, ion transport, molecular dynamics

\section{Introduction}

Traumatic brain injury (TBI) is a result of an insult to the brain caused by an external physical force, resulting in a range of neurological and psychological impairments. At the moment of injury, shearing and stretching forces can cause direct mechanical poration of neuronal membranes leading to altered membrane permeability[1-4]. This occurs through a cascade of events initiated through abnormal migration [2] of proteins and an influx of damaging ions[5] through the altered membranes. Ions such as potassium, sodium, calcium and chloride are a vital component in the human brain and are required for cell function, but a homoeostatic imbalance can lead to irreversible damage. For instance, sodium ion homeostasis is a major process in cells through coupled exchange with potassium ions between intra- and extracellular spaces using the sodium-potassium pump[6]. An increased influx of sodium ions into the cell would cause membrane depolarization and allow for a large increase in the release of glutamate into the extracellular space[7] leading to calcium influx through the ion pore of the receptor. This influx can result in disruption of calcium homeostasis, which is a key contributor to cell damage and death. Since the net influx of ions is much greater than the net efflux, an osmotic force is generated which drives an influx of water. This leads to axonal swelling and cytotoxic edema of glial cells which is tied to cell death[8].

Even so, there is potential for cellular repair and resealing over time resulting in cellular recovery. The pores that are observed in the membrane differ substantially in size and depth, and they evolve with time. The resealing time of these pores and the associated mechanisms depend strongly on its size and physico-chemical context[9]. Membrane resealing has been observed within seconds to minutes in non-neuronal cells [10-13] and within minutes to hours in neuronal cells [14-16] after mechanical injury. Although these works on neuronal cells suggest that 
neuronal membrane resealing takes minutes to hours, another experimental study suggests that some resealing could occur immediately [1]. The authors showed that a fraction of neurons could reseal early in post injury, but then may suffer secondary membrane reopening[1]. The relationship between mechanical deformation and changes in cellular permeability as a function of time before and after mechanical stretch has also been highlighted in neuronal cells[17]. During this study, the number and size of the molecules able to permeate through the cellular membrane decreased as a function of time suggesting cellular restoration.

Pore sizes produced through mechanoporation can be obtained by studying primary cortical neurons in in vitro models of neuronal stretch. To this end, TBI cellular models have been developed to understand the effects of biomechanical loading conditions at the cellular level[17,18]. For instance, Geddes et al.[17] assessed the size and duration of transient pores in a membrane following stretch by evaluating the uptake of a series of fluorescent molecules and fluorescently conjugated dextrans of various molecular masses. The authors found that immediately after insult moderate stress pores had a radii between $0.7 \mathrm{~nm}$ and $2.7 \mathrm{~nm}$, and they suggested that $2 \mathrm{~nm}$ pores may result in cellular damage and death in TBI. A similar pore size of around $2.7 \mathrm{~nm}$ was also observed through other in vivo studies[1,19].

The effects of mechanical stress on pore formation have been investigated through stretching simulations at atomistic[20,21] and coarse-grained[22] resolutions. Coarse-grained simulations of spherical cellular membranes subjected to shock have been performed[23], where pressure impulses were shown to cause pore formation in the lipid membrane. Due to limitations of the coarse-grained model, it was not possible to consider the transport of specific ion species through the pores, and there were restrictions on the pore sizes due to the spatial resolution of the model. To further elucidate the mechanism of ion transport through pores that can be formed through impact with a shock wave, simulations at the resolution of atoms must be employed. Leontiadou et al. studied the stability and the diffusion of sodium and chloride ions through tension stabilized water pores using atomistic simulations, where they observed cation-specific to anion-specific permeation with increasing pore radius[26,27]. Diffusion of ions associated with sodium chloride and potassium chloride driven by a transmembrane density gradient of cations were considered separately by Gurtovenko et al[28], where the stability of pores was also studied.

In this work, we use all-atom molecular dynamics (MD) simulations to study potassium, sodium, and chloride ions transport through transmembrane pores. Pores of radius 1.0, 1.5 and 2 $\mathrm{nm}$ were considered. We do not apply tension to stabilize the pore. Instead, we allow the pore to close in real time. We monitor the time-dependent ion transport through a pore that connects an extra- and intra-cellular compartment to further understand the damage threshold that can lead to a change of ion homeostasis after brain trauma. This ion-transport is driven by an ion imbalance between the two regions, where the concentration of ions in the intra- and extra-cellular membrane is similar to those found in neurological cells.

\section{Model and Methods}

\section{Membrane Model}

Cell membranes are complex, inhomogeneous structures that consist of multiple lipids, cholesterol, and numerous proteins. These structural complexities makes simulation of a realistic cell membrane a challenging task, so a simplified model is typically used. In this representation, 
only the major constituent of the cell membrane is considered: the phospholipid bilayer. Since the goal of this research is to study the mechanism resulting in chemical imbalance due to cellular damage associated with mechanoporation, it is not necessary to simulate the cell membrane in full detail.

Realistic ionic gradients and asymmetric salt concentrations across the neuronal cell membrane were considered in our simulations. MD simulations were performed on two membrane patches that separate the intracellular and extracellular space, where the solvent, lipids, and ions are represented in full atomistic detail[24]. The membrane patch was constructed using the membrane plugin in the Visual Molecular Dynamics (VMD) program [25]. The two lipid bilayers are both composed of 1-palmitoyl-2-oleoyl-sn-glycero-3-phosphocholine (POPC) lipids, which is appropriate for simulating a mammalian cell membrane system[26]. POPC lipids tend to make up the zwitterionic (uncharged) lipid component of a membrane, although most biological membranes are composed of a mixture of zwitterionic and anionic phospholipids. In neuronal cell membranes, the content of the neutral lipid is high compared to other biomembranes, where the anionic lipids only make up $\sim 10 \%$ by mass and are mainly located in the inner leaflets of the membrane[27].

A pore was incorporated into one of the lipid bilayers by removing lipids in the center of the bilayer up to the desirable pore radius. In this study, we considered pores that have an initial radius of 1.0, 1.5 and $2 \mathrm{~nm}$. Since pores can expand or shrink with time during the simulation, we monitor the number of water molecules in the pore central region. The pore size has been estimated using this quantity by Leontiadou et al.[28,29]. The interior region of the pore is defined as the region $\pm 0.75 \mathrm{~nm}$ from the bilayer center in the $z$ direction. The second bilayer was left intact (see Figure 1). The membrane is fully solvated using the solvation plug-in in VMD, where water molecules within the hydrophobic portion of the membrane were removed. The final system contains 495-535 POPC lipids and 37854 water molecules. Each bilayer has an area of $\sim 100 \times 100 \AA^{2}$.

Sodium, potassium, and chloride ions were included in the intra- and extra-cellular compartments by randomly substituting water molecules with the desired ions. An asymmetric ionic concentration was employed, where concentrations of each ion was chosen to mimic values found in neurological cells. We considered $\mathrm{Na}^{+}, \mathrm{K}^{+}$and $\mathrm{Cl}^{-}$concentrations of 145,3 , and 120 $\mathrm{mM}$ in the extracellular space, and 18,135 , and $7 \mathrm{mM}$ in the intracellular compartment, respectively[30]. Since the concentration of $\mathrm{Ca}^{2+}$ is low, 0.0001 and $1.2 \mathrm{mM}$ in the intra- and extra-cellular compartments, respectively, its presence is neglected in our simulations. In particular, we place 46 potassium, 6 sodium and 2 chloride ions in the intracellular compartment and 1 potassium, 49 sodium and 40 chloride ions in the extracellular space. When ions were added to each compartment according to a prescribed salt concentrations, the volume is only estimated from the number of water molecules in the system.

To neutralize the total charge, we add additional negative counter ions, namely 50 and 10 $\mathrm{Cl}^{-}$in the intra- and extra-cellular compartments, respectively. These additional chloride ions are kept constrained inside the intra- and extra- cellular compartment of the membrane patch to mimic fixed anions. In living cells, negatively charged organic macromolecules which are made up of mostly proteins are the major contributors of intercellular negatively charged ions. They are typically confined within the cells, and act as its fixed anions, where these anions contribute greatly to the intracellular osmolarity of the cell due to their large population. Although our model approximates these large molecules as spatially constrained negatively charged anions, 
this simplification still allows us to capture an osmotic influx of water, which we will discuss further.

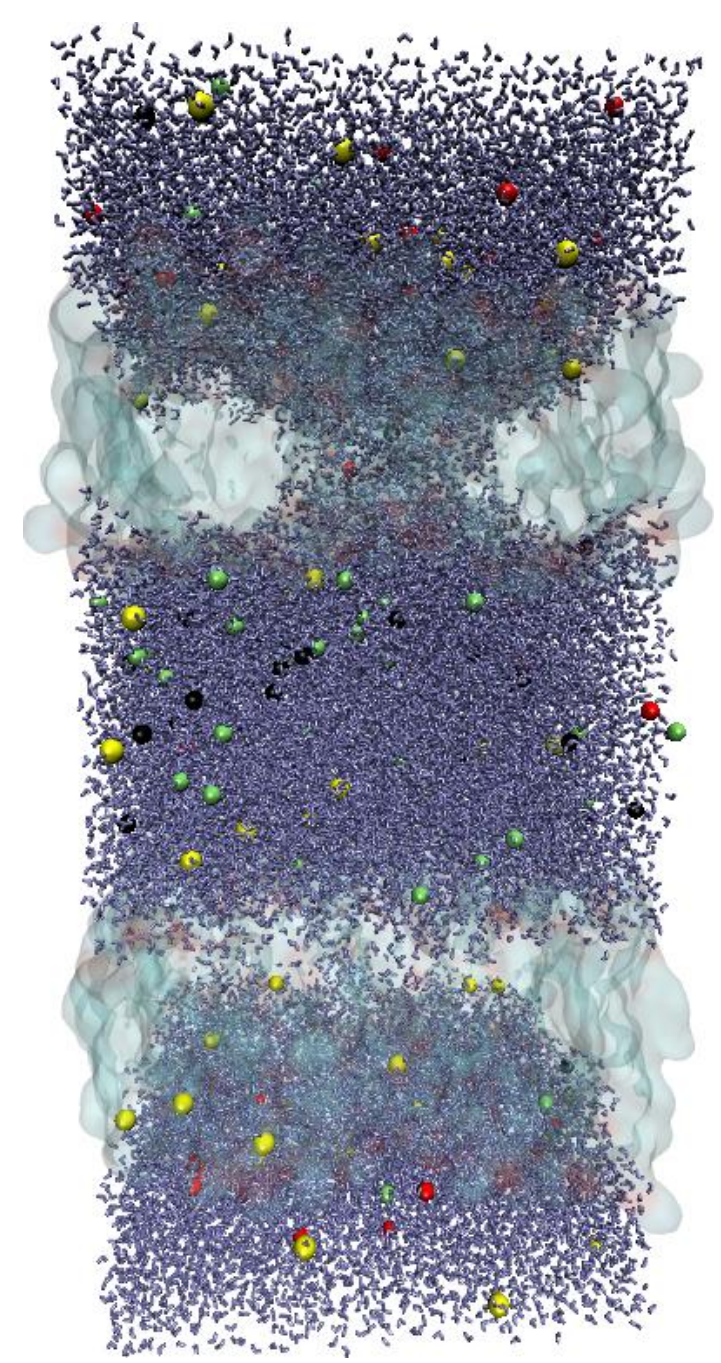

Figure 1. (color online) Representative snapshot of the simulation cell which consists of two membrane patches with intracellular compartment and extracellular space. The water molecules are displayed as blue and white sticks. Lipids are shown using a transparent space model. The potassium, sodium and chloride ions are shown as black, yellow and red spheres, respectively. The counter anions are shown with green spheres.

\section{Computational Methods}

Simulations were performed using the MD program NAMD (NAMD_CVS-2013-02-25), with the all-atom CHARMM36 lipid force field[31,32]. The TIP3P water model was used in CHARMM[33], where forcefield parameters for the ions were obtained from Ref.[34]. Electrostatic forces were evaluated using the particle mesh Ewald approach[35] and periodic boundary conditions were employed in all three dimensions. A time step of $1 \mathrm{fs}$ is used throughout the simulation. In the simulations mentioned below, constant pressure was maintained through the use of the Nosé-Hoover Langevin piston method, where we used a 200 
ps oscillation time and 50 ps of damping time constant to keep constant pressure. The target temperature was maintained through a Langevin thermostat with a damping coefficient of $1 \mathrm{ps}^{-1}$ for all of the atoms except for the ions. Three replicas were considered for each pore size.

After construction of the simulation cell described in the previous section, we initially performed a constant volume and temperature (NVT) simulation for $0.25 \mathrm{~ns}$, where all of the atoms except for the lipid tails were fixed. This was performed to introduce an appropriate amount of disorder in the bilayers. This was followed by a short equilibration of the system ( $0.25 \mathrm{~ns})$ under constant pressure and temperature (NPT), where we used anisotropic pressure coupling. Only a short equilibration is required since the distance between the layers was set to fit the actual membrane thickness, and the lattice period was set to fit the actual surface density of lipid molecules. The resulting area per lipid converges to a value of approximately $72 \AA^{2}$ per headgroup, which is similar to the experimental value of $68 \AA^{2}$ [36].

After this initial equilibration, simulations were performed under a pressure of 1.01325 bar and a temperature of $310 \mathrm{~K}$ under conditions of constant pressure, area, and temperature (NPAT). In these simulations, the cross-sectional area was kept constant. This simulation was performed for $135 \mathrm{~ns}$ or until the pore closed. To surmount finite size effects associated with the bilayers and the applied periodic boundary conditions, MD simulations of lipid bilayers are more consistently performed under the NPAT ensemble. Under this ensemble, small finite systems do not reproduce bilayer undulations, which would result in an underestimation of the area per lipid for simulations performed at zero surface tension. Although a modified version of the CHARMM36 force field was shown to reproduce the structural parameters of two bilayers at zero surface tension[37,38], the potential dependency of these parameters on system size is still a concern[39].

There are limitations with using MD simulations associated with timescale, where simulation times are typically not sufficient to observe many biological phenomena such as the dissipation of large pores. This makes it difficult to directly compare our computational results with experimental data. Conversely, experimentally it is very difficult to isolate microscopic mechanisms of pore stabilization and ion transport which occurs on the scale of nanoseconds after cellular injury. MD simulations can elucidate such mechanisms in isolation of competing molecular-scale factors, making simulations complementary to experimental efforts.

\section{Results}

\section{Pore Size and Stability}

Three initial pore radii $\left(\mathrm{r}_{\mathrm{i}}\right)$ were considered in this study: 1.0, 1.5 and $2 \mathrm{~nm}$. Similar pores have been considered in other computational studies [28] and observed experimentally[1,17,19]. To monitor the change in pore size, which decreases as a function of the entire simulation time, the number of water molecules, $N_{w}{ }^{p}$, and lipid head groups, $N_{h}{ }^{p}$, in the central pore region are monitored during the entire simulation for all three pore sizes. Since the pores were generated by removing lipids in the center of the bilayer, the initial pore surface is hydrophobic. During the simulation, the lipid headgroups start contouring the pore surface making it hydrophilic.

The smallest pore $\left(r_{i}=1 \mathrm{~nm}\right)$, was found to be unstable due to an insignificant hydrophilic lining (Figure $2 \mathrm{a}, \mathrm{b}$ ). The small pores close rapidly, where the closing time is less than $3.5 \mathrm{~ns}$ (Figure 2 a). During this time, no ion transport between the intra- and extra-cellular regions through the pore was detected. The brief lifetime of the pores could be attributed to an insufficient amount of stabilizing lipid lining $\left(N_{h}{ }^{p}<4\right)$, resulting in expulsion of water followed 
by pore closure. These results differ from the results presented by Leontiadou et al.[28,29], where the authors found that pores of $\mathrm{r}_{\mathrm{i}}=0.8 \mathrm{~nm}$ are stable. This disparity could arise from the different methodology that is used in our simulation study. Unlike the previous study, our pores are not stabilized by applying an external surface tension[28,29]. Since we are interested in the damage threshold leading to ionic disturbances after brain trauma, we allow the pore to close in real time.
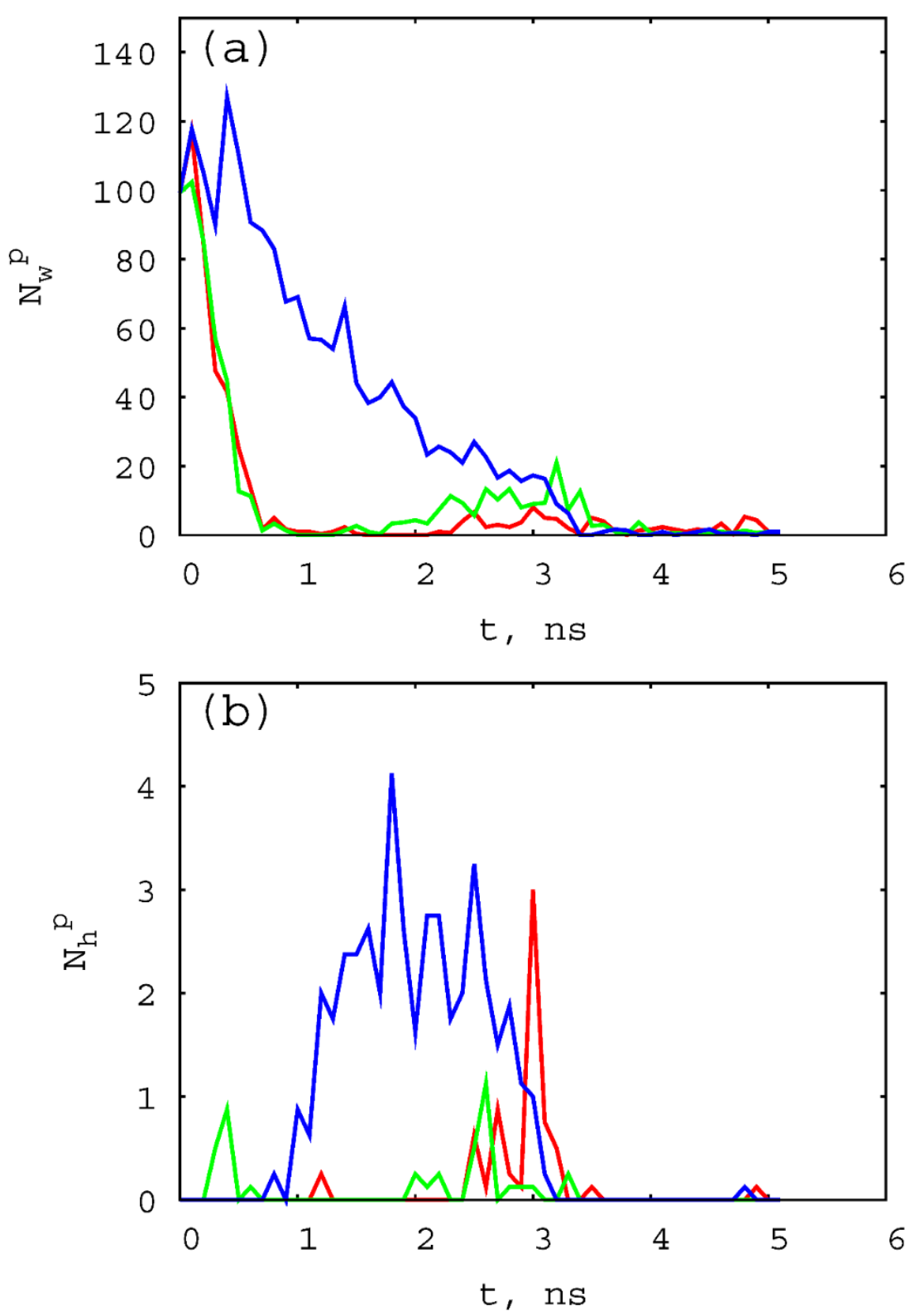

Figure 2. (color online) (a) Change of size of the small pore $\left(\mathrm{r}_{\mathrm{i}}=1 \mathrm{~nm}\right)$ vs time, $t$ measured as the number of water molecules in the pore, $N_{w}{ }^{p}$, (b) Number of lipid heads in the pore, $N_{h}{ }^{p}$ vs time, $t$. The three initial conditions are shown with different colors on the plots. 
The medium size pores $\left(r_{i}=1.5 \mathrm{~nm}\right)$ also close during the course of the simulation, but the resealing occurs over a longer time scale as shown in Figure 3a. Consequently, some degree of ion transport through the pore is possible as we will discuss in a later section. These medium pores are stable for around 10-60 ns, where we observe a dependency between the pore closure and the amount of hydrophilic pore lining (Figure 3). In particular, the resealing time appears to depend on the number of lipids that make up the hydrophilic lining of the pore (Figure $3 \mathrm{~b}$ ). In the three replicas, one sample did not have a sufficient amount of stabilizing lipid lining $\left(N_{h}^{p} \sim 15\right)$, which results in rapid pore closure at $\sim 20$ ns (Figure 3, green). The other two replicas, which consist of a comparable amount of pore lining $\left(N_{h}^{p} \sim 30\right)$ exhibit similar behavior as a function of time, and exhibit closure at a much later time, $\sim 100 \mathrm{~ns}$.
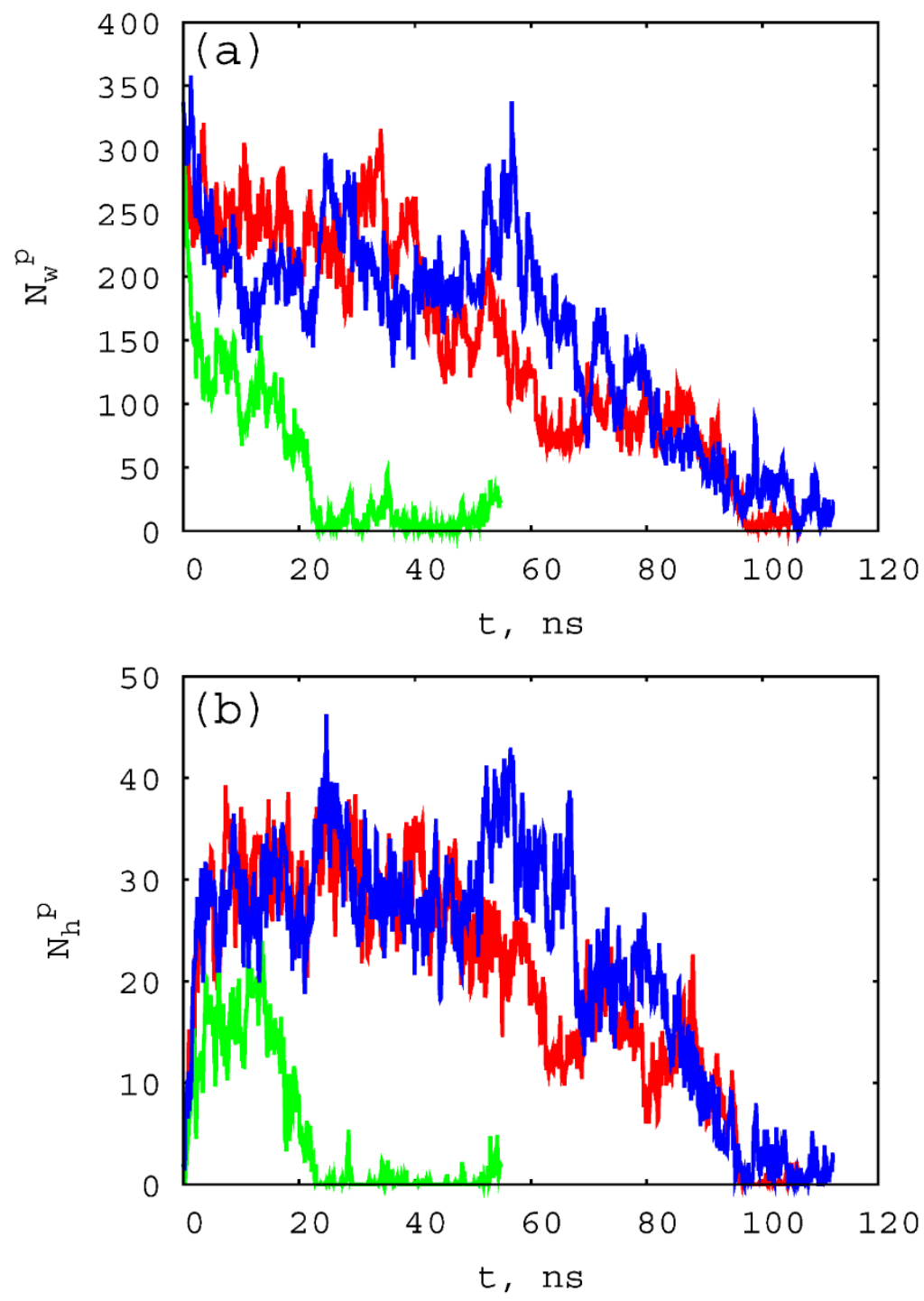

Figure 3. (color online) (a) Change of size of the medium pore $\left(r_{i}=1.5 \mathrm{~nm}\right)$ vs time, $t$ measured as the number of water molecules in the pore, $N_{w}{ }^{p}$, (b) Number of lipid heads in the pore, $N_{h}{ }^{p}$ vs time, $t$. The three initial conditions are shown with different colors on the plots. 
The largest pores considered in our study $\left(\mathrm{r}_{\mathrm{i}}=2 \mathrm{~nm}\right)$ did not close prior to the end of the simulation, which accessed a timescale of $\sim 135 \mathrm{~ns}$ as shown in Figure 4 . The initial pores produced through the three replicas exhibit a similar number of lipid heads and water molecules. Thus, the profiles of $N_{w}{ }^{p}$ and $N_{h}{ }^{p}$ do not significantly differ for three runs. The number of lipids in the pore lining is sufficient $\left(N_{h}^{p} \sim 45\right)$ to keep the pore open during the course of the simulation. Although the pores remained open for the entire time of our simulation, the pores may exhibit resealing at longer time scales which can be on the order of minutes. This timescale is not feasible with all-atom MD simulations. Since larger pore sizes are expected to have a sufficient amount of pore lining, pores with radii larger than $2 \mathrm{~nm}$ are not expected to undergo the resealing process within the same timeframe.
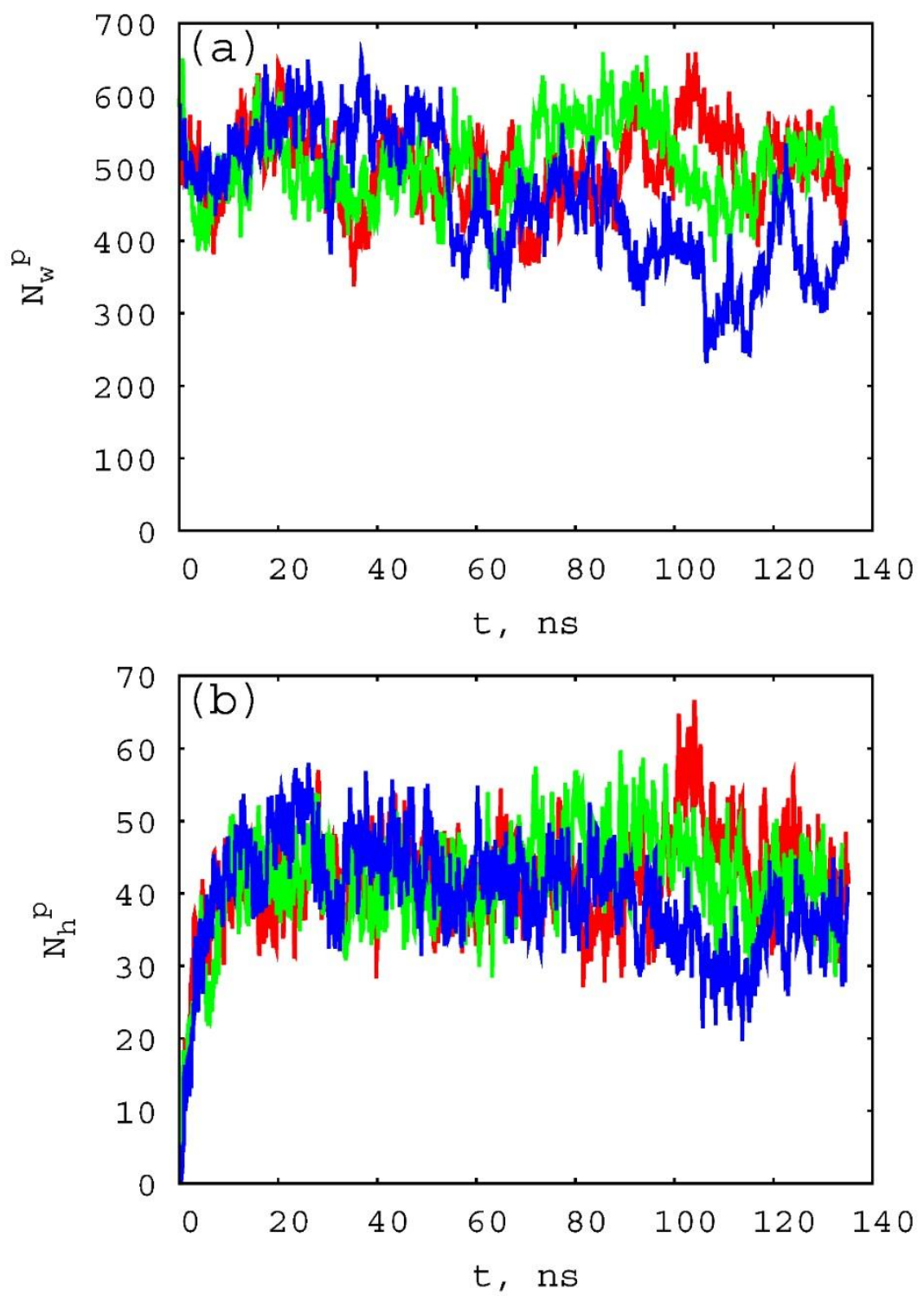
Figure 4. (color online) (a) Change of size of the large pore $\left(r_{i}=2 \mathrm{~nm}\right)$ vs time, $\mathrm{t}$ measured as the number of water molecules in the pore, $N_{w}{ }^{p}$, (b) Number of lipid heads in the pore, $N_{h}{ }^{p}$ vs time, $t$. The three initial conditions are shown with different colors on the plots.

\section{Ion Flux through the Pore}

Cell membranes form a barrier to various proteins, molecules, and ions dissolved in the intracellular and extracellular fluids. They are mostly impermeable, but the incorporation of a pore will facilitate ion diffusion, which is driven by an ion concentration gradient in the intraand extra-cellular compartments. In this section, we will monitor the change in ion concentration in the intracellular compartment of the membrane, and the ion influx through the pore for our two largest pore sizes. The smallest pore size $\left(r_{i=1} 1 \mathrm{~nm}\right)$ does not exhibit any ion transport prior to resealing.

Figure 5 illustrates the ion exchange through the medium and large pore throughout the course of the simulation, where the ion concentration for $\mathrm{K}^{+}, \mathrm{Na}^{+}$, and $\mathrm{Cl}^{-}$are monitored in the intracellular compartment. For the medium pore, the concentration does not change significantly and stops after $\sim 50 \mathrm{~ns}$ as result of pore resealing (Figure 5a), where the final intracellular concentrations of ions are $131 \mathrm{mM}, 23 \mathrm{mM}$, and $9 \mathrm{mM}$ for $\mathrm{K}^{+}, \mathrm{Na}^{+}$, and $\mathrm{Cl}^{-}$, respectively. In contrast, ion flux through the large pore is substantial, and a major change of ion concentration in the intracellular and extracellular compartments was observed (Figure 5b).

For a pore of $\mathrm{r}_{\mathrm{i}}=2 \mathrm{~nm}$, our simulation results show that the intracellular concentration of $\mathrm{Cl}^{-}$rises at a rate of $0.28 \mathrm{mM} / \mathrm{ns}$ for the first $100 \mathrm{~ns}$ of the run. After $\sim 100 \mathrm{~ns}$, the transport of $\mathrm{Cl}^{-}$ nearly ceases, where the intracellular concentration of $\mathrm{Cl}^{-}$reaches a new stable value of $\sim 32.5$ $\mathrm{mM}$. Similarly, the intracellular concentration of $\mathrm{Na}^{+}$also increases linearly for the first $100 \mathrm{~ns}$ though at a higher rate of $0.39 \mathrm{mM} / \mathrm{ns}$. After $100 \mathrm{~ns}$, the rate of change of the intracellular concentration of $\mathrm{Na}^{+}$decreases to $0.16 \mathrm{mM} / \mathrm{ns}$. On the other hand, the concentration of $\mathrm{K}^{+}$in the intracellular space decreases at a rate of $0.18 \mathrm{mM} / \mathrm{ns}$ for the entire run of $135 \mathrm{~ns}$. Thus the influx of the sodium ions through the membrane is initially faster than of the efflux of the potassium ions. After $\sim 100 \mathrm{~ns}$, when the intracellular concentration of $\mathrm{Cl}^{-}$levels off, the rate of influx of $\mathrm{Na}^{+}$becomes equal to the rate of efflux of $\mathrm{K}^{+}$(see Figure $5 \mathrm{~b}$ ). Our results show that the net charge is close to zero in both the intracellular and extracellular compartments during the entire duration of the simulation. Similar behavior is also expected to be observed within our simulation time frame for larger pore sizes. 

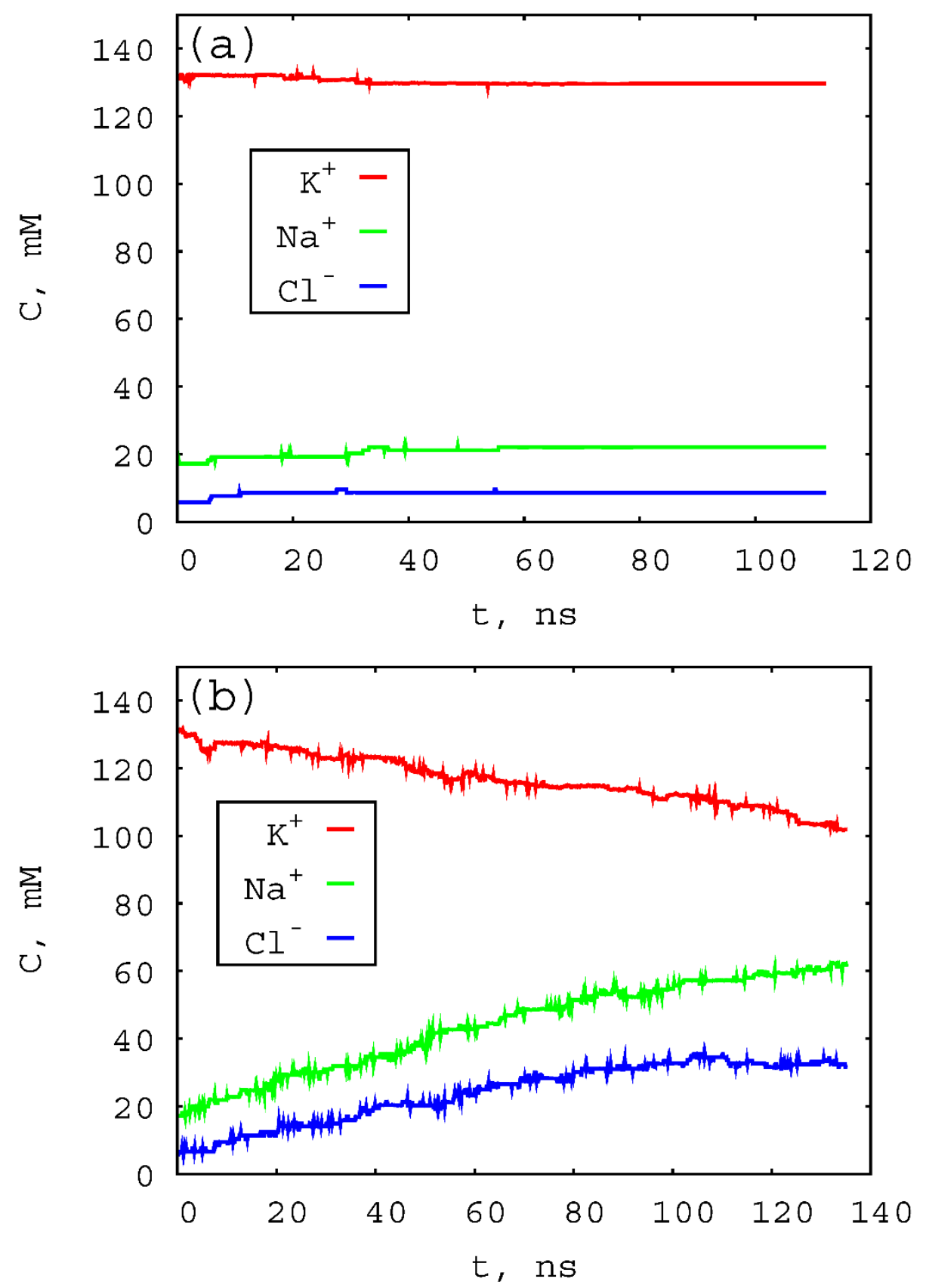

Figure 5. (color online) Change of ion concentration, $C$ with time, $t$ inside of the intracellular compartment (a) for the medium pore $\left(\mathrm{r}_{\mathrm{i}}=1.5 \mathrm{~nm}\right)$. (b) for the large pore $\left(\underline{r}_{i \underline{ }} 2 \mathrm{~nm}\right)$. The results are an average of three independent runs.

Initially the total concentration of ions, which consist of $\mathrm{Na}^{+}, \mathrm{K}^{+}$and $\mathrm{Cl}^{-}$, is higher in the extracellular space than in the intracellular compartment (the constrained anions are not included in this consideration). For the large pore, the ions appear to move through the two cell compartments according to their concentration gradient to balance the ion concentration in the 
intra- and extra-cellular compartments. Since the volume of both compartments are almost identical, we plotted the total number of ions located in the intracellular and the extracellular compartments, respectively (see Figure 6a). This analysis was only performed for the system with the largest pore, because the number of ions in both compartments changes only slightly, if at all, for the pores of smaller radius $\left(\mathrm{r}_{\mathrm{i}}<2 \mathrm{~nm}\right)$. When the concentration of the ions inside and outside of the cell levels off, ion flow between the two compartments slows down. This is expected since the concentration gradient is no longer driving ion transport between the two compartments through the pore. This could be attributed to a new resting state where solutes are at equilibrium across the membrane, which we would also expect to observe within our $135 \mathrm{~ns}$ simulation time for pores with $\mathrm{r}_{\mathrm{i}}>2.0 \mathrm{~nm}$.

As mentioned earlier, the intra-cellular compartment of the cell is in reality full of negatively charged proteins and other macromolecules which tend to bind to $\mathrm{K}^{+}$, resulting in a substantially lower efflux of $\mathrm{K}^{+}$relative to influx of $\mathrm{Na}^{+}[40]$. In our simplified model, fixed anions within the intra-cellular compartment approximates the role of these negatively charged molecules, where we observe similar behavior in the flux of $\mathrm{Na}^{+}$and $\mathrm{K}^{+}$through the pore (Figure $6 b)$. Unfixed $\mathrm{Cl}^{-}$also move between the two compartments to maintain an approximate net charge of zero in each compartment. Our results indicate that transport of $\mathrm{Cl}^{-}$is stopped after $\sim 100 \mathrm{~ns}$, when the new resting state is reached. This results in a reduction in the $\mathrm{Na}^{+}$inflow to the intracellular space and the rate of influx of $\mathrm{Na}^{+}$becomes equal to the rate of efflux of $\mathrm{K}^{+}$as mentioned earlier.

Our model membrane does not consider the anionic lipids present in cell-membranes[27], where it is expected that more cations would undergo adsorption in the mixed zwitterionic/anionic system than in the pure POPC bilayers therefore hindering their dynamics/mobility. Although, we did not include anionic lipids, we did add additional negative counter ions inside the intra- and extra- cellular compartments of the membrane patch to mimic fixed anions. Because of this, the omission of anionic lipids is not expected to dramatically influence our results. In the future we plan to include these lipids and their effect on ionic transfer for these membranes. 

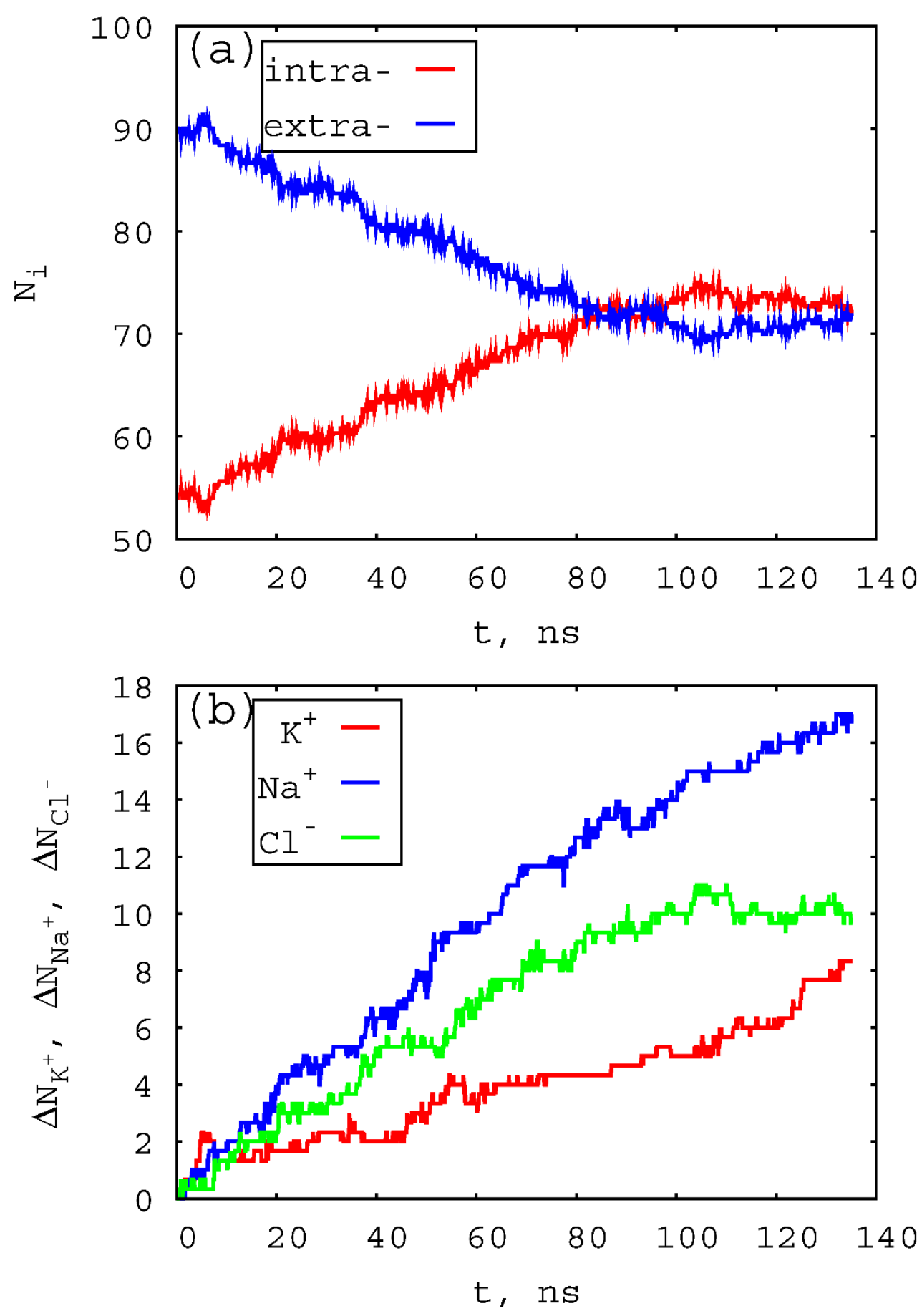

Figure 6. (color online) Ion influx through a membrane containing a large pore $\left(\mathrm{r}_{\mathrm{i}}=2 \mathrm{~nm}\right)$. (a) The number of ions in the inter- and extra-cellular compartment, $N_{i}$ as a function of time, $t$. (b) $\mathrm{K}^{+}$efflux, $\Delta N_{K}^{+}, \mathrm{Na}^{+}$ influx, $\Delta N_{N a}^{+}$and $\mathrm{Cl}^{-}$influx, $\Delta N_{C l}$, measured as a change of the respective number of ions in the extraand intercellular compartments, respectively. The results are an average of three independent runs.

Without a countervailing mechanism, this net inflow of $\mathrm{Na}^{+}$and $\mathrm{Cl}^{-}$, will lead to an increase of the cytosolic (intra-cellular) solute concentration. This could result in the rupture of the cell membrane due to the osmotic force that is generated by the influx of water into the 
cell[40,41]. This influx of water into the cell was also observed in our simulation as shown in Figure 7. In this plot, we computed the number of water molecules in the intracellular compartment for the large pore as a function of time, where the amount of water was found to increase on average by $\sim 9$ molecules per ns. The number of water molecules in the intra-cellular compartment increases by $\sim 5.5 \%$ during the course of the 135 ns simulation.

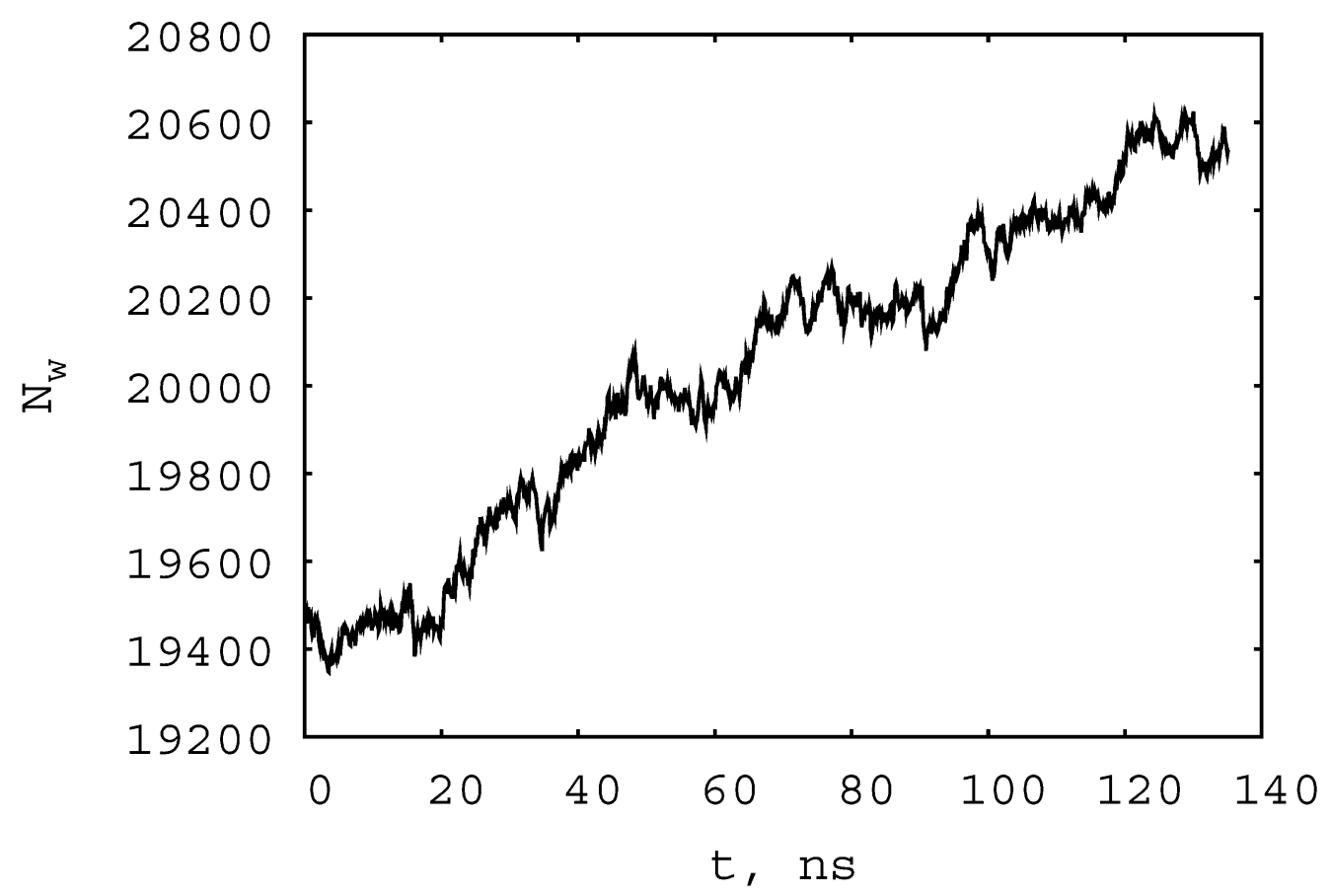

Figure 7. Number of water molecules in the intracellular compartment, $N_{w}$ as a function of time, $t$, for a membrane containing a large pore $\left(\mathrm{r}_{\mathrm{i}}=2 \mathrm{~nm}\right)$. The results are an average of three independent runs.

These results suggest that even relatively small pores of at least $r_{i}=2 \mathrm{~nm}$ can affect the homeostasis within a damaged cell within a relatively short time scale. In addition, these pores show a significant influx of water into the cell which could lead to cell rupture if the pore does not exhibit resealing at longer time scales. Smaller pores with $\mathrm{r}_{\mathrm{i}} \leq 1.5 \mathrm{~nm}$ exhibit resealing and do not significantly affect the resting membrane potential.

\section{Conclusion}

We employed all-atom MD simulations to study potassium, sodium, and chloride ions transport through transmembrane pores between the extra- and intra-cellular compartments. The concentration gradient between the two regions is the driving force for ions transport between the two compartments via the transmembrane pore. The goal of this work was to systematically evaluate the mechanism of membrane resealing and the effect of these transient pores on membrane permeability. To find a damage threshold that leads to a change of ion homeostasis after brain trauma, membrane transport and concentration were monitored as a function of time for three pore sizes: $\mathrm{r}_{\mathrm{i}}=1.0,1.5$ and $2 \mathrm{~nm}$. Pores around these sizes have been observed experimentally[1,17,19]. Our simulations showed that pores with a radius of $1.5 \mathrm{~nm}$ or less exhibited resealing over the course of our $\sim 135 \mathrm{~ns}$ simulations. Ionic disturbance was minimal at 
$\mathrm{r}_{\mathrm{i}}=1.5 \mathrm{~nm}$ and nonexistent at $\mathrm{r}_{\mathrm{i}}=1.0 \mathrm{~nm}$. Ion flux through the largest pore led to a substantial change in the intra- and extra-cellular ionic concentrations, and the system reached a new resting state after $\sim 100 \mathrm{~ns}$. The simulations showed that the influx of sodium and chloride ions was greater than efflux of potassium ions, which consequently caused an osmotic influx of water.

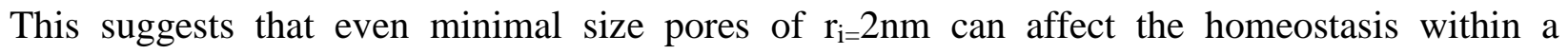
damaged cell within a relatively short time scale. Smaller pores do not significantly affect the resting potential due to resealing at timescales less than $\sim 100 \mathrm{~ns}$. Even though exact correspondence in time and length scales between simulation and experiment cannot be expected because of the small size and idealized representation of the simulation membrane compared to experiment, insight into the immediate effect of ion transport and how it relates to the structure of the pores is achieved through this effort. In addition, some of the findings for $r_{i}=2.0 \mathrm{~nm}$ may be applicable to larger pores. We expect that larger pores will remain open for a longer time that will be sufficient to reach a new resting state.

\section{Acknowledgements}

The authors would like to thank Drs. R. M. Elder, T. Piehler, J. Vettel, and P. Franaszczuk for useful discussion. Calculations were performed using the DOD Supercomputing Resource Center located at the Air Force and Navy Laboratories. The research reported in this document was performed in connection with contract/instrument W911-QX-14-C0016 with the U.S. Army Research Laboratory. The views and conclusions contained in this document are those of TKC Global Inc. and the U.S. Army Research Laboratory. Citation of manufacturer's or trade names does not constitute an official endorsement or approval of the use thereof. The U.S. Government is authorized to reproduce and distribute reprints for Government purposes notwithstanding any copyright notation hereon.

\section{References}

[1] O. Farkas, J. Lifshitz, J.T. Povlishock, The Journal of neuroscience : the official journal of the Society for Neuroscience 26 (2006) 3130.

[2] P. Arun, R. Abu-Taleb, S. Oguntayo, M. Tanaka, Y. Wang, M. Valiyaveettil, J.B. Long, Y. Zhang, M.P. Nambiar, Neuroscience letters 552 (2013) 87.

[3] M. Tymianski, Adv. Neurol. 71 (1996) 85.

[4] J.T. Weber, Frontiers in pharmacology 3 (2012) 60.

[5] R.H. Singleton, J.T. Povlishock, The Journal of neuroscience : the official journal of the Society for Neuroscience 24 (2004) 3543.

[6] A.M. Rose, R. Valdes Jr., Clin. Chem. 40 (1994) 1674.

[7] J.M. Hinzman, T.C. Thomas, J.J. Burmeister, J.E. Quintero, P. Huettl, F. Pomerleau, G.A. Gerhardt, J. Lifshitz, J Neurotrauma 27 (2010) 889.

[8] M.D. Tang-Schomer, V.E. Johnson, P.W. Baas, W. Stewart, D.H. Smith, Experimental neurology 233 (2012) 364.

[9] A.J. Jimenez, F. Perez, Seminars in cell \& developmental biology 45 (2015) 2.

[10] P.L. McNeil, S.S. Vogel, K. Miyake, M. Terasaki, Journal of Cell Science 113 (2000) 1891.

[11] M. Terasaki, K. Miyake, P.L. McNeil, The Journal of Cell Biology 139 (1997) 63.

[12] P.L. McNeil, S. Ito, Gastroenterology 96 (1989) 1238.

[13] P.L. NcNeil, R. Khakee, American Journal of Pathology 140 (1992) 1097. 
[14] H. Yawo, M. Kuno, The Journal of Neuroscience 5 (1985) 1626.

[15] R. Shi, J.D. Pryor, Neuroscience 98 (2000) 157.

[16] R. Shi, X. Qiao, N. Emerson, A. Malcom, J. Neurocytol. 30 (2001) 829.

[17] D.M. Geddes, R.S. Cargill II, M.C. LaPlaca, J Neurotrauma 20 (2003) 1039.

[18] M. Skotak, F. Wang, N. Chandra, Journal of neuroscience methods 205 (2012) 159.

[19] P.R. Williams, B.N. Marincu, C.D. Sorbara, C.F. Mahler, A.M. Schumacher, O. Griesbeck, M. Kerschensteiner, T. Misgeld, Nature communications 5 (2014) 5683.

[20] K. Koshiyama, S. Wada, Journal of biomechanics 44 (2011) 2053.

[21] T. Shigematsu, K. Koshiyama, S. Wada, Chemistry and physics of lipids 183 (2014) 43.

[22] T.V. Tolpekina, W.K. den Otter, W.J. Briels, The Journal of chemical physics 121 (2004) 8014.

[23] Y. Sliozberg, T. Chantawansri, The Journal of chemical physics 141 (2014) 184904.

[24] A.A. Gurtovenko, The Journal of chemical physics 122 (2005) 244902.

[25] W. Humphrey, A. Dalke, K. Schulten, J. Molec. Graphics 14 (1996) 33.

[26] P.C. Lai, C.J. Crasto, Front Genet. 3 (2012) 61.

[27] P.T. Wong, Ph. D, University of Michigan, Michigan, 2009.

[28] H. Leontiadou, A.E. Mark, S.J. Marrink, Biophysical journal 92 (2007) 4209.

[29] H. Leontiadou, A.E. Mark, S.J. Marrink, Biophysical journal 66 (2004) 2156.

[30] S. Squire, B. Darwin, F. Bloom, S. du Lac, A. Ghosh, Fundamental Neuroscience, Elsevier, Burlington, MA, 2008.

[31] J.B. Klauda, R.M. Venable, J.A. Freites, J.W. O'Connor, D.J. Tobias, C. MondragonRamirez, I. Vorobyov, A.D. MacKerell, Jr., R.W. Pastor, The journal of physical chemistry. B 114 (2010) 7830.

[32] J.B. Klauda, V. Monje, T. Kim, W. Im, J. Phys. Chem. B 116 (2012) 9424.

[33] W.L. Jorgensen, J. Chandrasekhar, J.D. Madura, R.W. Impey, M.L. Klein, J. Chem. Phys. 79 (1983) 926.

[34] D. Beglov, B. Roux, J. Chem. Phys. 100 (1994) 9050.

[35] P. Ewald, Ann Phys. 64 (1921) 253.

[36] N. Kucerka, J.D. Perlmutter, J. Pan, S. Tristram-Nagle, J. Katsaras, J.N. Sachs, Biophysical journal 95 (2008) 2792.

[37] J. Sonne, M.O. Jensen, F.Y. Hansen, L. Hemmingsen, G.H. Peters, Biophysical journal 92 (2007) 4157.

[38] C.-J. Högberg, A.P. Lyubartsev, Biophysical journal 94 (2008) 525.

[39] T. Broemstrup, N. Reuter, Biophysical journal 99 (2010) 825.

[40] J.M. Simard, T.A. Kent, M. Chen, K.V. Tarasov, V. Gerzanich, The Lancet Neurology 6 (2007) 258.

[41] H. Lodish, A. Berk, S.L. Zipurskey, P. Matsudaira, D. Baltimore, J. Darnell, Molecular Cell Biology, Freeman, New York, 2000. 